\title{
Generation of internal tides in an ocean of finite depth: analytical and numerical calculations
}

\author{
S. Khatiwala* \\ Department of Earth, Atmospheric and Planetary Sciences, Massachusetts Institute of Technology, Cambridge, MA 02139, USA
}

Received 20 July 2001; received in revised form 11 April 2002; accepted 25 September 2002

\begin{abstract}
Mixing in the abyssal ocean is known to play an important role in controlling the large-scale ocean circulation. In the search for sources of mechanical energy for mixing, internal tides generated by the interaction of the barotropic tide with bottom topography (mode conversion) have been implicated. However, estimates of the rate at which barotropic tidal energy is converted into the internal wave field are quite uncertain. Here, I present analytical and numerical calculations of internal tide generation in a fluid layer of finite depth to better understand the energetics of the wave generation process. Previous theoretical models of wave generation have assumed an upper radiation boundary condition (BC) appropriate for an ocean of infinite depth. But recent observations of internal tides at significant distances from their generation region indicate that this $\mathrm{BC}$ is not always valid, and that reflection from the upper surface is important. I show that the presence of an upper free-surface reduces the rate at which energy is fed into the internal wave field (the power) and thus the energy available for mixing. This reduction increases with the horizontal extent of the topography (relative to the wavelength of a mode-1 internal wave). Fully nonhydrostatic, nonlinear numerical calculations are used to both test the theory and to explore more realistic parameters for which linear theories are formally invalid. As bottom topography becomes steeper, linear theory underestimates mode conversion by an increasing amount, although even at critical slope the difference is quite small $(\mathrm{O}(20 \%))$. An important finding of this study is that for certain topographic shapes the power input into the wave field can saturate as the topography becomes supercritical. A comparison of model results with a recent finite amplitude theory suggests that even though finite depth effects may be negligible in the linear regime, they may become important when the topography is of finite amplitude. The results of process studies such as this should lead to improved estimates of mode conversion in the ocean.
\end{abstract} (C) 2003 Elsevier Science Ltd. All rights reserved.

\section{Introduction}

The subject of diapycnal mixing in the abyssal ocean has received much attention in recent years.

\footnotetext{
*Corresponding author. Present address: Lamont-Doherty Earth Observatory, Columbia University, Oceanography 201, Palisades, NY 10964, USA. Fax: + 1-845-365-8736.

E-mail address: spk@1deo.columbia.edu (S. Khatiwala).
}

Munk and Wunsch (1998) have discussed its importance in driving the meridional overturning circulation. They estimate the power required to return dense deep water, convectively formed at high-latitudes, back to the surface as $\sim 2.1 \mathrm{TW}$, and suggest that the mechanical energy required to accomplish this could come only from the winds or the tides. Winds can perhaps contribute $\sim 1 \mathrm{TW}$ (Wunsch, 1998). Tidal dissipation is known to be 
$\sim 3.7 \mathrm{TW}$ (2.5 TW from the semi-diurnal tide, $\mathbf{M}_{2}$ ), but much of this dissipation has traditionally been viewed as taking place in the bottom boundary layers of marginal seas (see Munk, 1997, for a review). The Munk and Wunsch (1998) estimate implies an average diapycnal diffusivity, $\kappa$, of about $10^{-4} \mathrm{~m}^{2} \mathrm{~s}^{-1}$ which has been difficult to reconcile observationally; measurements of the internal wave field (an important source of energy for mixing) imply a diffusivity of $\mathrm{O}\left(10^{-5} \mathrm{~m}^{2} \mathrm{~s}^{-1}\right)$. However, recent observations by Polzin et al. (1997) and Ledwell et al. (2000) based on measurements of microstructure and tracer dispersion convincingly show that diapycnal mixing (and the implied diffusivity) is strongly enhanced over rough bottom topography. They proposed that the enhanced mixing above rough topography was associated with conversion of barotropic tidal energy into baroclinic tidal energy (termed "mode conversion" by Munk and Wunsch, 1998), and the subsequent instability of the waves leading to breaking. These results are supported by more indirect inferences based on comparing numerical simulations of the barotropic tide with altimeter observations of sea-surface height (Egbert and Ray, 2000; Jayne and St. Laurent, 2001), suggesting that about $30 \%$ of tidal dissipation $(\sim 1 \mathrm{TW})$ takes place in the open ocean. However, since we do not have reliable estimates of the rate at which tidal energy is converted into the internal wave field, it is not clear to what extent the mechanism of mode conversion can contribute to the required dissipation of $2.1 \mathrm{TW}$.

In this paper, I examine the problem of internal wave generation by the interaction of an oscillating barotropic tidal flow with bottom topography in a fluid of finite depth. I focus on the energetics of the problem and present analytical expressions for the rate at which energy is input to the internal wave field (power). The power is related to the horizontal flux of energy away from the generation site and is a measure of the energy available for mixing. The theoretical predictions are tested against explicit numerical calculations of the wave generation process in a nonhydrostatic, nonlinear, hydrodynamic model. The numerical calculations also allow me to examine regimes where the theory is not formally valid.
In the next section, I briefly review existing models of internal tide generation.

\section{Wave generation models}

A number of analytical models of internal tide generation exist. These models, which are all linear in the dynamics, have been used to predict the energy input into the internal tides by a barotropic tide interacting with bottom topography. For conditions when the steepness parameter, $\varepsilon$, defined as the ratio of the topographic slope to the ray slope (see Section 4.2), is less than one (subcritical topography), models based on ray tracing such as those developed by Baines (1973, 1982) are available. These models, which are difficult to apply except for special topographic shapes, do not include advection by the background flow, but impose the bottom boundary condition (BC) at $z=h(x)$.

When $\varepsilon$ is much less than 1, Bell's (1975a, 1975b) analysis has been widely applied (for e.g., St. Laurent and Garrett, 2002; Polzin, submitted for publication). Bell's model linearizes the bottom condition by applying it at $z=0$ rather than at the bottom topography $z=h(x)$. The theory can be applied to arbitrary bottom topography. A crucial element of this model is the inclusion of advection by the background flow, which, as discussed in detail by Polzin (submitted for publication), is important for any realistic characterization of dissipation. Essentially, mid-ocean ridge bathymetry can be described as fractal, that is, the topographic slope variance is unbounded as smaller and smaller scales are included in the slope estimate. Polzin argues that a model which neglects advection will predict a large, small-scale response (the shear spectrum is "blue"). As a consequence, the integrated (over all wavenumbers) vertical shear and energy are both infinite. In contrast, incorporating the effect of advection has the effect of maintaining a finite shear variance at high horizontal wavenumbers, with associated implications for studies of mixing where an accurate specification of the vertical shear in the wave field is essential.

The wave generation model to be presented below is in part based on the work of Bell (1975a). 
My analysis too is applicable to "weak" topography $(\varepsilon \ll 1)$, but with an important modification. Bell's theory is applicable for a fluid of infinite depth in which energy input at the bottom can radiate upward. Bell (1975b) argued that an upper radiation condition was justified because most of the energy traveling upward would be dissipated through various processes, including shear instability and wave-wave interactions (which transfer energy to smaller scales). In the ocean, however, it is not clear what fraction of the internal wave energy generated at the ocean bottom survives to reflect off the free-surface or for that matter make multiple reflections between the bottom and surface. For instance, St. Laurent and Garrett (2002) have suggested that the Richardson number of the internal tide is not small enough to cause shear instability. Furthermore, they contend that the time scale for wavewave interaction is long relative to the propagation time of the internal tide. Citing the work of Ray and Mitchum (1996), who observed low-mode internal tides at distances of $\mathrm{O}(1000 \mathrm{~km})$ from their generation site (the Hawaiian Ridge), they conclude that dissipation is small enough for the energy to undergo reflections from the free-surface and the bottom. Dushaw et al. (1995) have also found a significant internal tide signal in reciprocal acoustic transmissions approximately $2000 \mathrm{~km}$ north of the Hawaiian Islands. According to them the likely source of these internal tides is the Hawaiian Ridge. Thus, there is compelling evidence to suggest that a radiation condition may not be the appropriate upper $\mathrm{BC}$, so in contrast to Bell, I will impose an upper rigid lid BC. An important goal of this paper is to explore the consequences of this upper rigid-lid $\mathrm{BC}$ for mode conversion. As will be shown below, this simple change in $\mathrm{BC}$ not only makes the problem mathematically more complex, but drastically alters the energetics of the wave generation process. In particular, the inclusion of an upper reflecting surface can greatly reduce, relative to the infinite-depth solution, the power input to the wave field and thus the energy available for mixing. It is thus not only of theoretical interest to inquire into the effect of such a $\mathrm{BC}$ on the wave generation process, but the results are also potentially very relevant to quantifying tidal dissipation in the ocean via mode conversion.

Two other studies that treat the finite-depth case should be mentioned. Hibiya (1986) has examined the transient problem of internal waves generated by flow over topography of an impulsively started mean flow in a fluid of finite depth, but the mathematical formulation of the problem as well as the results and focus of that paper are fundamentally very different from the present work. In particular, Hibiya (1986) does not consider the steady state response to an oscillating tidal mean flow or the energetics of the wave generation process as is done here. After the present study was completed, I became aware of a recent paper by Llewellyn Smith and Young (2002) submitted for publication. They too present an analytical treatment of the problem of internal tide generation in a fluid of finite depth (although their mathematical approach is quite different), and highlight the role of an upper $\mathrm{BC}$ in reducing barotropic to baroclinic mode conversion. Unlike the present study, however, they do not compare their results with numerical calculations.

Some of the results to be presented here can be anticipated from the simpler theory of lee waves, which is briefly reviewed in the next section.

\section{Steady background flow}

It is useful to begin with the problem of internal gravity waves generated by a steady mean flow over topography. I will use this mathematically simpler and better known setting of lee wave generation to illustrate how different the wave generation problem is in the presence of an upper "rigid lid" BC (as would be appropriate for a fluid layer of finite depth) compared with the situation when an upper "radiation" condition is imposed (as in an infinitely deep fluid layer). Analytic solutions will be derived for both periodic and arbitrary topography.

\subsection{Periodic topography}

Consider a steady, background flow, $U$, over periodic topography, $h(x)=h_{0} \cos k x$ (Fig. 1). For 


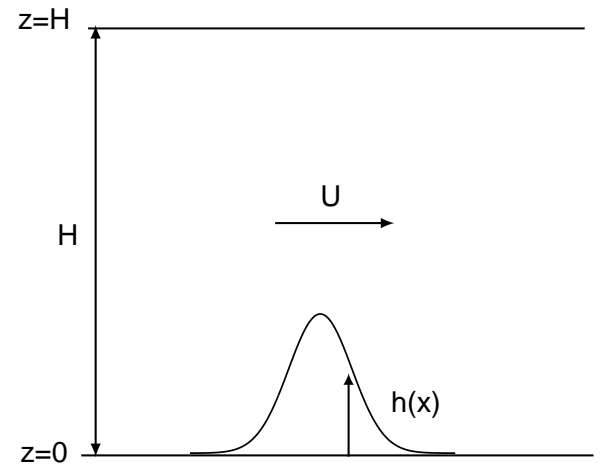

Fig. 1. Schematic used to illustrate the situation being modeled and to establish notation.

two-dimensional $(x, z)$ disturbances, the linearized equations for momentum and buoyancy can be combined to form a single equation for vorticity,

$\nabla^{2} \psi+\frac{N^{2}}{U^{2}} \psi=0$

where $N$ is the (constant) buoyancy frequency, and the streamfunction, $\psi$, is defined by

$u^{\prime}=-\partial \psi / \partial z, \quad w^{\prime}=\partial \psi / \partial x$.

$u^{\prime}$ and $w^{\prime}$ are the horizontal and vertical velocity components of the disturbance. For now we ignore the influence of rotation. The lower BC, applied in the linear theory at $z=0$, is

$$
\begin{aligned}
w^{\prime}(x, z=0) & =U \frac{\partial h}{\partial x} \\
\Rightarrow \psi(x, z & =0)=U h(x) .
\end{aligned}
$$

Substituting a separable solution of the form $\psi(x, z)=\hat{\psi}(z) \mathrm{e}^{\mathrm{i} k x}$

(where the real part is implied) into Eq. (1) gives

$\frac{\mathrm{d}^{2} \hat{\psi}}{\mathrm{d} z^{2}}+\left(\frac{N^{2}}{U^{2}}-k^{2}\right) \hat{\psi}=0$.

The bottom BC, Eq. (2), becomes $\hat{\psi}(0)=U h_{0}$. We now contrast the two situations of interest.

Case I: Upper radiation boundary condition. Depending on the values of $N$ and $U$ there are two possibilities. When $\mu^{2} \equiv k^{2}-N^{2} / U^{2}>0$, the medium cannot sustain internal waves (the intrinsic frequency, $U k$, is larger than $N$ ). The appropriate upper $\mathrm{BC}$ is that the disturbance decays with height which then gives

$\psi=U h_{0} \mathrm{e}^{-\mu z} \cos k x, \quad \mu=\left(k^{2}-\frac{N^{2}}{U^{2}}\right)^{1 / 2}$.

From the momentum equation, the perturbation pressure, $p^{\prime}$, is given by

$p^{\prime}=\rho_{0} U \partial \psi / \partial z$.

Eq. (4) then implies that the lows and highs of the perturbation pressure coincide with the troughs and crests of the topography and thus the force per unit area on the topography

$F=\frac{k}{2 \pi} \int_{0}^{2 \pi / k} p^{\prime}(x, z=0) \frac{\mathrm{d} h}{\mathrm{~d} x} \mathrm{~d} x$

vanishes. Note that there is an equal and opposite force exerted on the fluid (the drag) which, here, is also zero. Equivalently, the vertical velocity, $w^{\prime}$, is $90^{\circ}$ out of phase with $p^{\prime}$, and so the vertical energy flux $\overline{p^{\prime} w^{\prime}}=\rho_{0} U \overline{\psi_{x} \psi_{z}}=0$ (where overbars represent a horizontal average over a wavelength $2 \pi / k$ ).

When $\mu^{2} \equiv N^{2} / U^{2}-k^{2}>0$, Eq. (3) admits wave solutions of the form $\hat{\psi} \sim \operatorname{expi} \mu^{ \pm} z$, where $\mu^{ \pm}= \pm \sqrt{N^{2} / U^{2}-k^{2}}$. A radiation condition must then be imposed to pick the correct solution, i.e., the energy flux is required to be outward as $z \rightarrow \infty\left(\overline{p^{\prime} w^{\prime}}>0\right)$. This condition can be shown to be equivalent to the requirement $\operatorname{sgn} \mu=\operatorname{sgn} k$. Assuming $k>0$, the solution is then

$\psi=U h_{0} \cos \left(k x+\mu^{+} z\right)$.

The pressure field is now such that there is a net force (Eq. (6)) exerted on the topography given by $F=\frac{1}{2} \rho_{0} U^{2} h_{0}^{2} \mu^{+} k>0$.

The rate at which work is done on the fluid is simply $-F U(<0)$, i.e., energy is removed from the background flow at lower levels to generate internal waves. The internal waves radiate this energy upward at a rate per unit area

$\overline{p^{\prime} w^{\prime}}=\frac{1}{2} \rho_{0} U^{3} h_{0}^{2} k \sqrt{N^{2} / U^{2}-k^{2}}$.

Case II: Upper rigid lid boundary condition. In the presence of an upper rigid lid, the $\mathrm{BC}$ at $z=H$ is

$$
\begin{gathered}
w^{\prime}(x, z=H)=0 \\
\Rightarrow \hat{\psi}(H)=0 .
\end{gathered}
$$


The solution is then $\psi=\hat{\psi} \cos k x$ with

$\hat{\psi}= \begin{cases}U h_{0} \frac{\sinh \mu(H-z)}{\sinh \mu H}, & \text { if } \mu^{2} \equiv k^{2}-\frac{N^{2}}{U^{2}}>0 \\ U h_{0} \frac{\sin \mu(H-z)}{\sin \mu H}, & \text { if } \mu^{2} \equiv \frac{N^{2}}{U^{2}}-k^{2}>0 .\end{cases}$

Eqs. (5) and (6) then imply that the drag is once again zero. The presence of an upper reflecting boundary (and a periodic topography) traps the energy in the vertical. This establishes a pressure field resulting in zero net force on the topography; in steady state, and in this inviscid system, no energy is converted from the mean flow into internal waves. This absence of wave drag when an upper reflecting condition is imposed is in sharp contrast to the presence of drag when an upper radiation condition is imposed.

\subsection{Arbitrary topography}

Let us now examine the problem of internal wave generation by a steady background flow over arbitrary topography, $h(x)$ (Fig. 1). The infinitedepth solution is simply a superposition (over all wavenumbers, $k$ ) of the solutions derived above for periodic topography and will not be discussed further. The finite-depth case is more interesting. Introducing the Fourier transform,

$\hat{f}(k)=\int_{-\infty}^{\infty} f(x) \mathrm{e}^{-\mathrm{i} k x} \mathrm{~d} x$,

the solution to Eq. (1) with BC Eqs. (2) and (7) is $\psi=\frac{1}{2 \pi} \int_{-\infty}^{\infty} \hat{\psi}(k, z) \mathrm{e}^{\mathrm{i} k x} \mathrm{~d} k$

where

$$
\begin{aligned}
& \hat{\psi}(k, z)= \\
& \left\{\begin{array}{cl}
U \hat{h}(k) \frac{\sinh \mu(H-z)}{\sinh \mu(k) H}, & \text { if } \mu^{2} \equiv k^{2}-\frac{N^{2}}{U^{2}}>0 \\
U \hat{h}(k) \frac{\sin \mu(H-z)}{\sin \mu(k) H}, & \text { if } \mu^{2} \equiv \frac{N^{2}}{U^{2}}-k^{2}>0 .
\end{array}\right.
\end{aligned}
$$

Invoking the two function version of Parseval's theorem, the net force on the topography can be written as

$$
\begin{aligned}
F & =\int_{-\infty}^{\infty} p^{\prime}(z=0) \frac{\mathrm{d} h}{\mathrm{~d} x} \mathrm{~d} x \\
& =\frac{1}{2 \pi} \int_{-\infty}^{\infty}(\mathrm{i} k \hat{h})^{*} \hat{p}^{\prime} \mathrm{d} k,
\end{aligned}
$$

where * denotes complex conjugation. From Eq. (5) it follows that

$$
F \approx \frac{\mathrm{i} \rho_{0} U^{2}}{2 \pi} \int_{-\infty}^{\infty}|\hat{h}(k)|^{2} \mu(k) k \frac{\cos \mu(k) H}{\sin \mu(k) H} \mathrm{~d} k .
$$

The integrand in Eq. (11) has singularities when the condition $\mu(k) H=j \pi$ ( $j$ an integer) is met. This condition implies that the poles of the integrand are located at $k= \pm k_{j}$,

$k_{j} \equiv\left(\frac{N^{2}}{U^{2}}-\frac{j^{2} \pi^{2}}{H^{2}}\right)^{1 / 2}, \quad j=1,2, \ldots$.

The "resonant" values of the vertical wave number $\mu=j \pi / H$ correspond to the normal modes of a resting layer of fluid with a flat bottom (e.g., Gill, 1982). Consideration of the initial value problem McIntyre, 1972; Baines, 1995) (or equivalently addition of friction to the steady problem; see below) shows that the contour of integration must be indented below the poles and, by Jordan's lemma (e.g., Carrier et al., 1966), closed in the upper half of the complex $k$-plane. By Cauchy's residue theorem we have

$F=2 \pi \mathrm{i} \sum$ residues enclosed.

Evaluating the residues by Taylor expanding the denominator of Eq. (9) about $k= \pm k_{j}$, the drag is found to be

$F=2 \frac{\rho_{0} U^{2}}{H^{3}} \sum_{j=1}^{j_{\max }} j^{2} \pi^{2}\left|\hat{h}\left(k_{j}\right)\right|^{2}$,

where $j_{\max }$ is the largest integer less than $N H / U \pi$ (an inverse Froude number). It is important to note that all the contributions to the force and hence the energy flux into the wave field $(F U)$ come from the "resonant" wavenumbers given by Eq. (12). As will be shown in the next section, this is also the case for an oscillating background flow. 


\section{Oscillating background flow}

The simple analytic solutions for a steady mean flow discussed above clearly illustrate the impact of an upper $\mathrm{BC}$ on the energetics of the wave field. In this section, we will examine the more complex problem of internal wave generation by the interaction of an oscillating background flow (the barotropic tide) with bottom topography in the presence of rotation. The resulting internal tide radiates energy away from the topography and into the ocean interior. I will present solutions for this energy flux by computing the rate at which energy is converted from the oscillating background flow into internal waves in a fluid of finite depth. As mentioned above, Bell (1975a) has derived solutions for a fluid of infinite depth, and while my derivation is based in part on that earlier work, the presence of an upper reflecting surface fundamentally changes the nature of the solutions.

\subsection{Basic solution}

Consider small perturbations about a background flow $U(t)=U_{0} \cos \omega_{0} t$ (Fig. 2) on an $f$-plane in a uniformly stratified fluid of mean depth $H$. The linearized equations of motion for small perturbations from the background flow are

$\mathscr{D} u^{\prime}-f v^{\prime}=-\frac{1}{\rho_{0}} \frac{\partial p^{\prime}}{\partial x}-r u^{\prime}$,

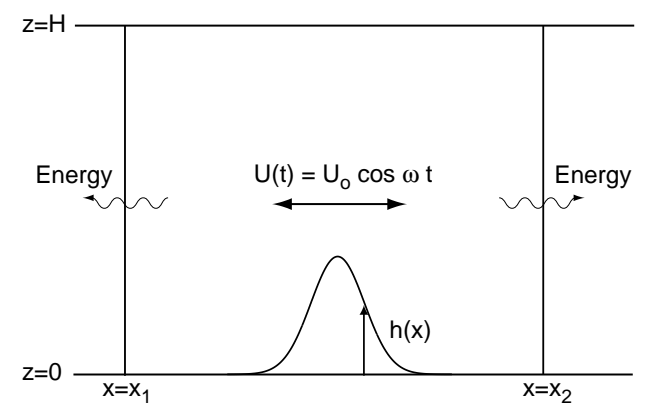

Fig. 2. Schematic to illustrate the generation of internal waves by an oscillating flow and the subsequent propagation of wave energy.

$$
\begin{aligned}
& \mathscr{D} v^{\prime}+f u^{\prime}=-\frac{1}{\rho_{0}} \frac{\partial p^{\prime}}{\partial y}-r v^{\prime}, \\
& \mathscr{D} w^{\prime}=-\frac{1}{\rho_{0}} \frac{\partial p^{\prime}}{\partial z}-b-r w^{\prime},
\end{aligned}
$$

where $u^{\prime}, v^{\prime}$, and $w^{\prime}$ are the zonal, meridional, and vertical components of the wave velocity, $b$ is the buoyancy, $f$ is the (constant) Coriolis parameter, and $r$ is an inverse time scale for damping. The operator $\mathscr{D}$ is defined by

$\mathscr{D}=\frac{\partial}{\partial t}+U(t) \frac{\partial}{\partial x}$.

The buoyancy $b$ is governed by

$\mathscr{D} b=N^{2} w^{\prime}$.

In this study, I am treating only the twodimensional situation, and will henceforth assume there is no $y$-dependence, that is, $\partial / \partial y \equiv 0$. (Since the topography is a function only of $x$, if the flow is initially $y$-independent, it will remain so. Furthermore, there is no inconsistency between assuming $y$ independence and including rotation $\left(v^{\prime}\right.$ is not zero).) The continuity equation then becomes

$\frac{\partial u^{\prime}}{\partial x}+\frac{\partial w^{\prime}}{\partial z}=0$.

The presence of dissipation in the momentum equations requires further explanation. As we will see, dissipative terms are introduced for purely mathematical reasons (to evaluate certain Fourier integrals). This is a standard mathematical expedient frequently invoked in many wave problems (e.g., Carrier et al., 1966). Indeed, the specific form of the dissipation (linear drag here) is immaterial, since we are interested in an inviscid theory, and the damping coefficient will eventually be set to zero.

In a frame of reference moving with the background flow, the governing equation for the vertical velocity, $W^{\prime}(\xi, z, t)$, as observed in the moving frame, is

$$
\begin{aligned}
\frac{\partial^{2}}{\partial t^{2}} \nabla^{2} W^{\prime}+f^{2} \frac{\partial^{2} W^{\prime}}{\partial z^{2}} & \\
& +N^{2} \frac{\partial^{2} W^{\prime}}{\partial \xi^{2}}+r \frac{\partial}{\partial t} \nabla^{2} W^{\prime}=0,
\end{aligned}
$$


where $\xi$ is the horizontal coordinate in the moving frame defined by

$$
\begin{aligned}
\xi(x, t) & =x-\int_{0}^{t} U_{0} \cos \omega_{0} t^{\prime} \mathrm{d} t^{\prime} \\
& =x-\frac{U_{0}}{\omega_{0}} \sin \omega_{0} t .
\end{aligned}
$$

The linearized lower $\mathrm{BC}$ on $W^{\prime}$ is

$$
W^{\prime}(\xi, z=0, t)=\frac{\partial h^{\prime}}{\partial t},
$$

where $h^{\prime}(\xi, t)$ is the height of the topography in the moving frame. The Fourier transform of $W^{\prime}$ is defined as

$$
\tilde{w}(k, z, t)=\int_{-\infty}^{\infty} W^{\prime}(\xi, z, t) \mathrm{e}^{-\mathrm{i} k \xi} \mathrm{d} \xi .
$$

The lower BC (Eq. (16)) implies

$\tilde{w}(k, 0, t)=\hat{h}(k) \sum_{n=-\infty}^{\infty} \operatorname{in} \omega_{0} J_{n}\left(\frac{U_{0} k}{\omega_{0}}\right) \mathrm{e}^{\mathrm{i} n \omega_{0} t}$,

where $\hat{h}(k)$ is the Fourier transform of the topography $h(x)$ and I have made use of the relation (Watson, 1966)

$\mathrm{e}^{\mathrm{i} z \sin \theta}=\sum_{n=-\infty}^{\infty} J_{n}(z) \mathrm{e}^{\mathrm{i} n \theta}$,

where $J_{n}$ is a Bessel function of order $n$. Following Bell (1975a), the BC Eq. (17) motivates us to seek a series solution of the form

$\tilde{w}(k, z, t)=\hat{h}(k) \sum_{n=-\infty}^{\infty} \tilde{W}_{n}(k, z) J_{n}\left(\frac{U_{0} k}{\omega_{0}}\right) \mathrm{e}^{\mathrm{i} n \omega_{0} t}$.

Fourier transforming Eq. (14) and substituting Eq. (18) we find

$$
\begin{aligned}
& \frac{\mathrm{d}^{2} \tilde{W}_{n}}{\mathrm{~d} z^{2}}+k^{2}\left[\frac{N^{2}-n^{2} \omega_{0}^{2}+\mathrm{i} r n \omega_{0}}{n^{2} \omega_{0}^{2}-f^{2}-\mathrm{i} r n \omega_{0}}\right] \tilde{W}_{n}=0 \\
& \tilde{W}_{n}(k, 0)=\mathrm{i} n \omega_{0}, \quad \tilde{W}_{n}(k, H)=0 .
\end{aligned}
$$

We will assume that $f<\omega_{0}$, which holds equatorward of about $75^{\circ}$ latitude if $\omega_{0}$ is the $\mathbf{M}_{2}$ frequency. Poleward of $75^{\circ}$ internal tides of this frequency are trapped. We will also restrict $n$ to integers $<n_{0}$, where $n_{0}$ is the maximum integer $<N / \omega_{0}$. The solution to the above equation is

$$
\tilde{W}_{n}(k, z)=\operatorname{in} \omega_{0} \frac{\sin \mu_{n}(k)(H-z)}{\sin \mu_{n}(k) H},
$$

where $\mu_{n}(k)$ is defined by

$\mu_{n}^{2}=k^{2}\left[\frac{N^{2}-n^{2} \omega_{0}^{2}+\mathrm{i} r n \omega_{0}}{n^{2} \omega_{0}^{2}-f^{2}-\mathrm{i} r n \omega_{0}}\right]$.

In the limit $r \rightarrow 0$ we wish to recover the positive root of $\mu_{n}$, and we therefore define $\mu_{n}$ as

$\mu_{n}(k) \equiv \operatorname{sgn}(\operatorname{Re} k) k\left[\frac{N^{2}-n^{2} \omega_{0}^{2}+\mathrm{i} r n \omega_{0}}{n^{2} \omega_{0}^{2}-f^{2}-\mathrm{i} r n \omega_{0}}\right]^{1 / 2}$,

where $k$ is now complex in general. The solution can then be written as

$w^{\prime}(x, z, t) \approx \sum_{\substack{n=-n_{0} \\(n \neq 0)}}^{n_{0}} \int_{-\infty}^{\infty} \hat{w}_{n}(k) \mathrm{d} k$,

where

$$
\begin{aligned}
\hat{w}_{n}(k)= & \frac{1}{2 \pi} \hat{h}(k) i n \omega_{0} \frac{\sin \mu_{n}(H-z)}{\sin \mu_{n} H} \\
& \times J_{n}\left(\frac{U_{0} k}{\omega_{0}}\right) \mathrm{e}^{\mathrm{i}\left(n \omega_{0} t+k \xi\right)}
\end{aligned}
$$

with $\xi$ defined in Eq. (15). From Eq. (21) we see that the integrand in Eq. (20) has singularities in the complex $k$-plane. The addition of friction has moved the poles off the $\operatorname{Re} k$ axis along which the integration (Eq. (20)) is carried out. The poles of the integrand satisfy $\mu_{n}(k) H=j \pi(j=1,2, \ldots)$, and are thus located at $k= \pm k_{j n}$, where

$k_{j n} \equiv \frac{j \pi}{H}\left[\frac{n^{2} \omega_{0}^{2}-f^{2}-\mathrm{i} r n \omega_{0}}{N^{2}-n^{2} \omega_{0}^{2}+\mathrm{i} r n \omega_{0}}\right]^{1 / 2}$.

It can be shown that $\operatorname{sgn}\left(\operatorname{Im} k_{j n}\right)=-\operatorname{sgn} n$. Fig. 3 shows the location of the poles in the complex $k$ plane. To evaluate the Fourier integral we apply Cauchy's theorem to the contours shown in Fig. 3. As is evident from Eq. (21), when $\xi=x-$ $\left(U_{0} / \omega_{0}\right) \sin \omega_{0} t>0$, the contour must be closed in the upper half of the complex plane along the path $C_{R}$ (Fig. 3) so that its contribution to the integral vanishes, as $R \rightarrow \infty$. Similarly, when $\xi<0$, the contour must be closed in the lower half of the complex plane along the path $C_{R^{\prime}}$. For brevity only the solution for $\xi>0$ will be presented in detail. In that case the contour encloses poles at $k=-k_{j n}$ if $n>0$ and at $k=+k_{j n}$ if $n<0$ (Fig. 3). Defining,

$R_{j n}^{-}=\operatorname{Residue}\left\{\hat{w}_{n}(k), k=-k_{j n}\right\}$,

$R_{j n}^{+}=\operatorname{Residue}\left\{\hat{w}_{n}(k), k=+k_{j n}\right\}$, 

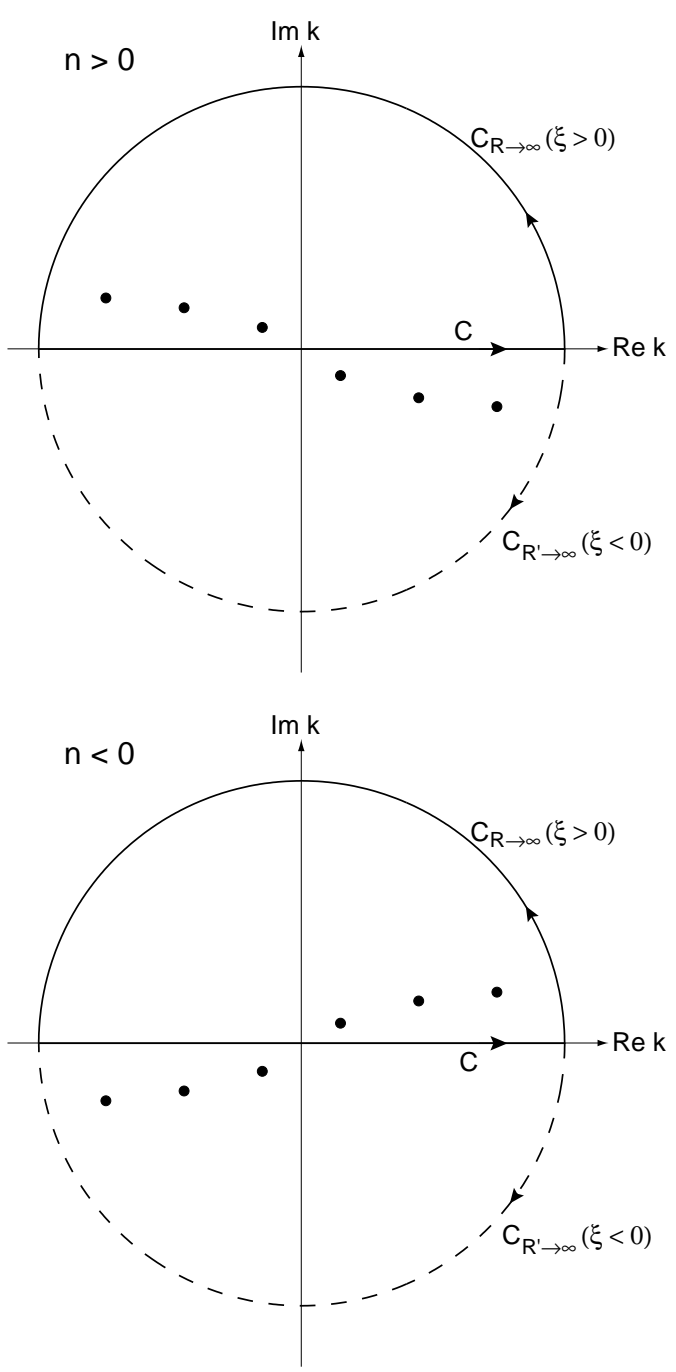

Fig. 3. Sketch of the complex $k$-plane showing location of poles $\pm k_{j n}(\bullet)$ when $n>0$ (top) and when $n<0$ (bottom). Additionally, when $\xi>0$ the contour is closed in the upper part of the complex plane $\left(C_{R}\right)$, and when $\xi<0$ it is closed in the lower half $\left(C_{R^{\prime}}\right)$.

Cauchy's theorem gives

$w^{\prime}(x, z, t) \approx 2 \pi \mathrm{i} \sum_{j=1}^{\infty}\left[\sum_{n=1}^{n_{0}} R_{j n}^{-}+\sum_{n=-n_{0}}^{-1} R_{j n}^{+}\right]$.

The residues are readily evaluated by Taylor expanding the denominator in Eq. (21) about $k= \pm k_{j n}$ to give

$$
\begin{aligned}
R_{j n}^{-}= & \frac{k_{j n}}{2 j \pi^{2}} \hat{h}\left(-k_{j n}\right) \mathrm{i} n \omega_{0} J_{n}\left(\frac{-U_{0} k_{j n}}{\omega_{0}}\right) \\
& \times \sin \frac{j \pi z}{H} \mathrm{e}^{\mathrm{i}\left(n \omega_{0} t-k_{j n} \xi\right)}, \\
R_{j n}^{+}= & -\frac{k_{j n}}{2 j \pi^{2}} \hat{h}\left(k_{j n}\right) \mathrm{i} n \omega_{0} J_{n}\left(\frac{U_{0} k_{j n}}{\omega_{0}}\right) \\
& \times \sin \frac{j \pi z}{H} \mathrm{e}^{\mathrm{i}\left(n \omega_{0} t+k_{j n} \xi\right)} .
\end{aligned}
$$

Taking the limit $r \rightarrow 0$ and making use of the relations (Watson, 1966)

$$
\begin{aligned}
& J_{-n}(z)=(-1)^{n} J_{n}(z), \\
& J_{n}(-z)=(-1)^{n} J_{n}(z),
\end{aligned}
$$

the various expressions can be combined to give the vertical velocity:

$$
\begin{aligned}
\xi>0 & : w^{\prime}(x, z, t) \\
\approx & \sum_{j=1}^{\infty}-\frac{1}{j \pi} \sum_{n=1}^{n_{0}}(-1)^{n} k_{j n} n \omega_{0} J_{n}\left(\frac{U_{0} k_{j n}}{\omega_{0}}\right) \sin \frac{j \pi z}{H} \\
& \times\left[\hat{h}\left(-k_{j n}\right) \mathrm{e}^{\mathrm{i}\left(n \omega_{0} t-k_{j n} \xi\right)}+\hat{h}\left(k_{j n}\right) \mathrm{e}^{-\mathrm{i}\left(n \omega_{0} t-k_{j n} \xi\right)}\right] \\
\xi<0: & w^{\prime}(x, z, t) \\
\approx & \sum_{j=1}^{\infty}-\frac{1}{j \pi} \sum_{n=1}^{n_{0}} k_{j n} n \omega_{0} J_{n}\left(\frac{U_{0} k_{j n}}{\omega_{0}}\right) \sin \frac{j \pi z}{H} \\
& \times\left[\hat{h}\left(k_{j n}\right) \mathrm{e}^{\mathrm{i}\left(n \omega_{0} t+k_{j n} \xi\right)}+\hat{h}\left(-k_{j n}\right) \mathrm{e}^{-\mathrm{i}\left(n \omega_{0} t+k_{j n} \xi\right)}\right],(23)
\end{aligned}
$$

where

$k_{j n}=\frac{j \pi}{H}\left[\frac{n^{2} \omega_{0}^{2}-f^{2}}{N^{2}-n^{2} \omega_{0}^{2}}\right]^{1 / 2}$.

Evidently, Eq. (23) consists of waves with a vertical modal structure propagating horizontally away from the obstacle.

\subsection{Conditions for validity of linear theory}

A number of assumptions were made in the derivation of the linear theory. In particular we required that both $u^{\prime}$ and $w^{\prime}$ were both $\ll U_{0}$. From the bottom BC, Eq. (2), $w^{\prime} \sim U_{0} h_{0} k$, where $h_{0}$ and $1 / k$ are characteristic vertical and horizontal scales, respectively, of the topography. Continuity then implies that $u^{\prime} k \sim w^{\prime} \mu$, or, $u^{\prime} \sim U_{0} h_{0} \mu$, where $\mu$ is a characteristic vertical wavenumber of the 
wave. Thus,

$$
\begin{aligned}
& u^{\prime} \ll U_{0} \Rightarrow h_{0} \mu \ll 1, \\
& w^{\prime} \ll U_{0} \Rightarrow h_{0} k \ll 1 .
\end{aligned}
$$

We examine the first of the above conditions more closely. There are two limiting cases. The quasistatic or lee wave regime is realized when $\omega_{0} / N \rightarrow 0$, and from Section 3 we recall that $\mu \sim N / U_{0}$. When $U_{0} k / \omega_{0} \rightarrow 0$, we have the acoustic limit (Bell, 1975a). In this limit, horizontal excursions by the background flow $\left(U_{0} / \omega_{0}\right)$ are much smaller than the length-scale of the topography. From Eq. (19), $\mu \sim N k / \omega_{0}$. Thus,

$h_{0} \mu \ll 1 \Rightarrow \begin{cases}N h_{0} / U_{0} \ll 1 & \text { quasi-static limit, } \\ N h_{0} k / \omega_{0} \ll 1 & \text { acoustic limit. }\end{cases}$

Defining a steepness parameter, $\varepsilon$,

$\varepsilon \equiv \frac{\text { topographic slope }}{\text { ray slope }}$

and recognizing that the ray slope, which is the angle of the wave characteristics, $\sim k / \mu$, we can conveniently summarize the above requirements by the single condition: $\varepsilon \ll 1$. In particular, I will define $\varepsilon$ as

$\varepsilon=\frac{h_{0} k}{\sqrt{\left(\omega_{0}^{2}-f^{2}\right) /\left(N^{2}-\omega_{0}^{2}\right)}}$.

When $\varepsilon<1$, the bottom topography is called subcritical, and when $\varepsilon>1$ it is characterized as being supercritical. The theory presented here is valid for $\varepsilon \ll 1$.

\subsection{Power input}

The pressure field associated with the internal wave solution derived above exerts a net force (Eq. (10)) on the bottom topography. As discussed in Section 3, this implies that net work is done by the background flow in generating the internal wave field. The rate of working is the power, $\mathscr{P}$. To compute it we need an expression for the force, $F$, and hence the pressure $p^{\prime}$. Eliminating $v^{\prime}$ from the horizontal momentum equations gives

$\mathscr{D}^{2} u^{\prime}+f^{2} u^{\prime}=-\frac{1}{\rho_{0}} \frac{\partial}{\partial x} \mathscr{D} p^{\prime}$.
Fourier transforming the above equation and the continuity equation results in

$\hat{\mathscr{D}}^{2} \hat{u}^{\prime}+f^{2} \hat{u}^{\prime}=-\frac{\mathrm{i} k}{\rho_{0}} \hat{\mathscr{D}} \hat{\hat{p}^{\prime}}$,

$\hat{u^{\prime}}=\frac{\mathrm{i}}{k} \frac{\partial \hat{w}^{\prime}}{\partial z}$,

where a ' denotes a Fourier transform (Eq. (8)) and $\hat{\mathscr{D}}=\partial / \partial t+\mathrm{i} k U(t)$. Solving for $\hat{p}^{\prime}$ :

$$
\begin{aligned}
\hat{p^{\prime}}= & -\rho_{0} \frac{\hat{h}(k)}{k^{2}} \sum_{\substack{n=-n_{0} \\
(n \neq 0)}}^{n_{0}} \mu_{n}(k)\left(n^{2} \omega_{0}^{2}-f^{2}\right) J_{n}\left(\frac{U_{0} k}{\omega_{0}}\right) \\
& \times \frac{\cos \mu_{n}(k)(H-z)}{\sin \mu_{n}(k) H} \mathrm{e}^{\mathrm{i}\left(n \omega_{0} t-\frac{U_{0} k}{\omega_{0}} \sin \omega_{0} t\right)},
\end{aligned}
$$

where $\mu_{n}$ is defined in Eq. (19). Employing Parseval's theorem (Eq. (10)) the force on the topography can be written as

$$
\begin{aligned}
F(t)= & \frac{\mathrm{i} \rho_{0}}{2 \pi} \sum_{\substack{n=-n_{0} \\
(n \neq 0)}}^{n_{0}} \int_{-\infty}^{\infty}|\hat{h}(k)|^{2} \frac{k^{*}}{k^{2}} \mu_{n}(k)\left(n^{2} \omega_{0}^{2}-f^{2}\right) \\
& \times J_{n}\left(\frac{U_{0} k}{\omega_{0}}\right) \frac{\cos \mu_{n}(k) H}{\sin \mu_{n}(k) H} \\
& \times \mathrm{e}^{\mathrm{i}\left(n \omega_{0} t-\left(U_{0} k / \omega_{0}\right) \sin \omega_{0} t\right)} \mathrm{d} k
\end{aligned}
$$

The integral above can be solved by the previous method, but it is more convenient to directly compute the power, which is defined as

$\mathscr{P}_{\mathrm{F}} \equiv \frac{\omega_{0}}{2 \pi} \int_{0}^{2 \pi / \omega_{0}} F(t) U_{0} \cos \omega_{0} t \mathrm{~d} t$.

$\mathscr{P}_{\mathrm{F}}$ is the rate (per unit cross-stream distance) at which energy is converted from the barotropic tide into the internal tide in a fluid of finite depth. From Watson (1966) we have the useful relation

$J_{n}(\eta)=\frac{\omega_{0}}{2 \pi} \int_{0}^{2 \pi / \omega_{0}} \mathrm{e}^{\mathrm{i}\left(n \omega_{0} t-\eta \sin \omega_{0} t\right)} \mathrm{d} t$

and hence

$$
\begin{aligned}
\mathscr{P}_{\mathrm{F}}= & \frac{\mathrm{i} \rho_{0}}{2 \pi} \sum_{\substack{n=-n_{0} \\
(n \neq 0)}}^{n_{0}} \int_{-\infty}^{\infty}|\hat{h}(k)|^{2} \frac{k^{*}}{k^{3}} \mu_{n}(k) n \omega_{0} \\
& \times\left(n^{2} \omega_{0}^{2}-f^{2}\right) J_{n}^{2}\left(\frac{U_{0} k}{\omega_{0}}\right) \frac{\cos \mu_{n}(k) H}{\sin \mu_{n}(k) H} \mathrm{~d} k .
\end{aligned}
$$

An application of Cauchy's theorem similar to that above finally gives the desired expression 
for $\mathscr{P}_{\mathrm{F}}$ :

$$
\begin{aligned}
\mathscr{P}_{\mathrm{F}}= & \frac{2 \rho_{0}}{\pi} \sum_{j=1}^{\infty} \sum_{n=1}^{n_{0}} \frac{\left|\hat{h}\left(k_{j n}\right)\right|^{2}}{j} J_{n}^{2}\left(\frac{U_{0} k_{j n}}{\omega_{0}}\right) \\
& \times n \omega_{0}\left(n^{2} \omega_{0}^{2}-f^{2}\right)^{1 / 2}\left(N^{2}-n^{2} \omega_{0}^{2}\right)^{1 / 2} .
\end{aligned}
$$

As in the lee wave problem, all the contributions to the power come from the resonant wavenumbers $k_{j n}$ (Eq. (24)). Thus for a "long" obstacle (i.e., one with a Fourier transform dominated by small wavenumbers) the power can vanish.

In a fluid of finite depth, the energy being fed into the internal wave field is trapped between the bottom and the free-surface at $z=H$, but can radiate horizontally away from the generation site. To better understand this, it is useful to construct an energy budget for the internal wave field. The wave energy density, $E$ (sum of kinetic and potential energy) is defined as

$E=\frac{\rho_{0}}{2}\left(u^{\prime 2}+v^{2}+w^{\prime 2}+N^{2} \eta^{2}\right)$,

where $\eta$ is the vertical displacement of fluid particles $\left(w^{\prime}=\mathscr{D} \eta\right.$ in a linear theory). The momentum and buoyancy equations can then be combined to form an energy equation:

$\mathscr{D} E=-\frac{\partial}{\partial x} p^{\prime} u^{\prime}-\frac{\partial}{\partial z} p^{\prime} w^{\prime}+q(x, z, t)$,

where $q$ is a source (or sink) of energy. Integrating the above equation vertically from $z=0$ to $H$, and horizontally from $x=x_{1}$ to $x_{2}$ (from one side of the obstacle to the other; see Fig. 2) the total energy in that region is governed by

$$
\begin{aligned}
& \frac{\partial}{\partial t} \int_{0}^{H} \int_{x_{1}}^{x_{2}} E(x, z, t) \mathrm{d} x \mathrm{~d} z \\
&=-U(t) \int_{0}^{H}\left[E\left(x_{2}, z, t\right)-E\left(x_{1}, z, t\right)\right] \mathrm{d} z \\
&-\int_{0}^{H}\left[\left.p^{\prime} u^{\prime}\right|_{x_{2}}-\left.p^{\prime} u^{\prime}\right|_{x_{1}}\right] \mathrm{d} z \\
&+\int_{0}^{H} \int_{x_{1}}^{x_{2}} q(x, z, t) \mathrm{d} x \mathrm{~d} z .
\end{aligned}
$$

Averaged over a period, the term on the LHS vanishes, while the final term on the RHS can be identified with the average energy input $\left(\mathscr{P}_{\mathrm{F}}\right)$ due to the work done by the background flow against the pressure force. (This last assertion can be demonstrated explicitly.) The energy relation then becomes

$$
\begin{aligned}
\mathscr{P}_{\mathrm{F}}= & \frac{\omega_{0}}{2 \pi} \int_{0}^{2 \pi / \omega_{0}} U(t) \\
& \times \int_{0}^{H}\left[E\left(x_{2}, z, t\right)-E\left(x_{1}, z, t\right)\right] \mathrm{d} z \mathrm{~d} t \\
& +\frac{\omega_{0}}{2 \pi} \int_{0}^{2 \pi / \omega_{0}} \int_{0}^{H}\left[\left.p^{\prime} u^{\prime}\right|_{x_{2}}-\left.p^{\prime} u^{\prime}\right|_{x_{1}}\right] \mathrm{d} z \mathrm{~d} t .
\end{aligned}
$$

The above expression says that the power input into the wave field is balanced by the horizontal flux of energy away from the obstacle (Fig. 2). This flux is due to advection by the background flow as well as by the pressure work (which dominates the flux).

\subsection{Comparison with the infinite-depth solution}

It is useful to compare the expression for power derived above (Eq. (28)) with Bell's solution (Bell, 1975a,b) for an infinitely deep fluid:

$$
\begin{aligned}
\mathscr{P}_{\infty}= & \frac{2 \rho_{0}}{\pi} \sum_{n=1}^{n_{0}} n \omega_{0}\left(n^{2} \omega_{0}^{2}-f^{2}\right)^{1 / 2}\left(N^{2}-n^{2} \omega_{0}^{2}\right)^{1 / 2} \\
& \times \int_{0}^{\infty} \frac{|\hat{h}(k)|^{2}}{k} J_{n}^{2}\left(\frac{U_{0} k}{\omega_{0}}\right) \mathrm{d} k .
\end{aligned}
$$

In an infinitely deep fluid, the power $\left(\mathscr{P}_{\infty}\right)$ can be related to the vertical flux of energy, $\overline{p^{\prime} w^{\prime}}$ : the power input at the bottom is radiated vertically upward. Note that Eq. (29) can be recovered as a limiting case of the finite-depth solution, Eq. (28), by letting the depth, $H$, of the fluid to go to infinity in the latter. To see this, we write

$k_{j n}=j \delta k_{n}$,

where

$\delta k_{n}=\frac{\pi}{H}\left[\frac{n^{2} \omega_{0}^{2}-f^{2}}{N^{2}-n^{2} \omega_{0}^{2}}\right]^{1 / 2}$.

In the limit $H \rightarrow \infty$, the spacing, $\delta k_{n}$, between the resonant wavenumbers goes to zero, and the sum (over $j$ ) in Eq. (28) can be replaced by an integral, and we recover the infinite-depth expression Eq. (29) $\left(1 / j=\delta k_{n} / k_{j n}\right)$.

To contrast the finite- and infinite-depth solutions, I will employ an idealized bottom 


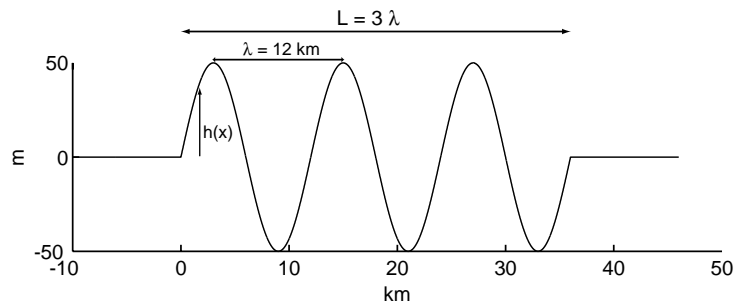

Fig. 4. Schematic of idealized topography used to illustrate the difference between a finite-depth and infinite-depth fluid. The truncated sinusoidal bathymetry shown here has $m=3$ wavelengths, so $L=3 \times \lambda=36 \mathrm{~km}$ The amplitude is $50 \mathrm{~m}$.

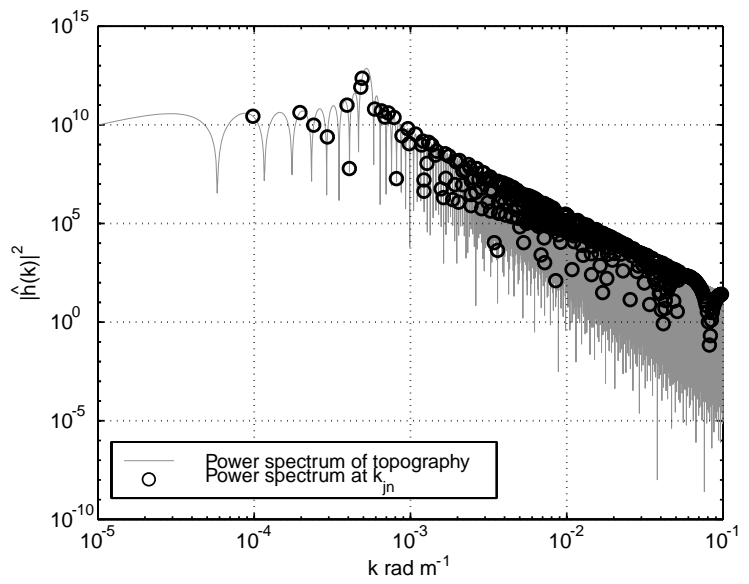

Fig. 5. Power spectrum $\left(|\hat{h}(k)|^{2}\right)$ (thin line) of the idealized sinusoidal topography with $m=9$ wavelengths $\left(L / \lambda_{1} \approx 1.7\right)$. The amplitude is $50 \mathrm{~m}$. Also shown (circles) is the power spectrum at wavenumbers $k_{j n}$ which contribute to the power in the finite-depth solution.

topography represented by a truncated sine wave (Fig. 4) consisting of an integer, $m$, number of wavelengths $(\lambda=12 \mathrm{~km})$. The bottom is flat on either side of this truncated sine, which has an amplitude of $50 \mathrm{~m}$. For reasons that will become clear, I will characterize this topography by $L \equiv$ $m \lambda$. For reference, Fig. 5 shows the power spectrum, $|\hat{h}(k)|^{2}$, of the topography for $m=9$. Also shown (circles) is the power spectrum evaluated at the discrete resonant wavenumbers, $k_{j n}$, which contribute to the power in the finitedepth theory. The nominal depth of the fluid is $H=4700 \mathrm{~m}$, basic state stratification is

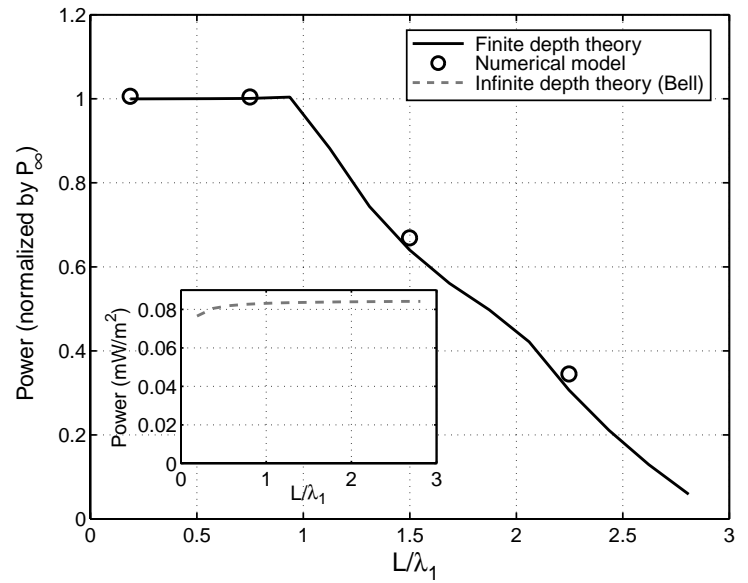

Fig. 6. Power input to the internal wave field by the interaction of an oscillating background flow with topography as a function of $L / \lambda_{1}$. Solid line is the power input, $\mathscr{P}_{\mathrm{F}}$ (normalized by $\mathscr{P}_{\infty}$ ) for a finite-depth fluid. Circles are results from numerical calculations of the internal tide (Section 2). Inset: absolute power input (per unit area) predicted by Bell's theory for an infinitely deep fluid.

$N=8 \times 10^{-4} \mathrm{~s}^{-1}$, and the Coriolis parameter is $f=8 \times 10^{-5} \mathrm{~s}^{-1}$. The assumed background flow has an amplitude $U_{0}=2 \mathrm{~cm} \mathrm{~s}^{-1}$ and oscillates in time at the $\mathrm{M}_{2}$ frequency $\left(\omega_{0}=1.4 \times 10^{-4} \mathrm{~s}^{-1}\right)$. These values are representative of open ocean conditions. For these parameter values, $U_{0} / \omega_{0} \lambda$, which is a measure of advection by the background flow relative to the length scale of the topography, is $\approx 0.01$, and the steepness parameter, $\varepsilon \approx 0.18$. We now examine how the power input, $\mathscr{P}_{\mathrm{F}}$ and $\mathscr{P}_{\infty}$, vary as a function of the ratio $L / \lambda_{1}$, where $\lambda_{1}(\approx 65 \mathrm{~km}$, here) is the wavelength of the mode-1 wave of the internal tide, i.e., $2 \pi / k_{11}$.

Fig. 6 shows the finite-depth mode conversion rate, normalized by the corresponding infinitedepth value (i.e., $\mathscr{P}_{\mathrm{F}} / \mathscr{P}_{\infty}$ ), as a function of $L / \lambda_{1}$. The inset shows the absolute infinite-depth power input per unit area $\left(\mathscr{P}_{\infty} / L\right)$. Physically, isolated topographic features (for e.g., seamounts) would be characterized by small values of $L / \lambda_{1}$, while more aerially extended features such as mid-ocean ridges would be represented by large values. As the ratio $L / \lambda_{1}$ increases the power input predicted by infinite-depth theory (inset) remains roughly 
constant. In contrast, in a finite-depth ocean, the predicted power input rapidly decreases. This result can be readily understood if we recall the results of Section 3. For a fluid of finite depth, it was shown that in the presence of a periodic bottom topography, the power input is exactly zero. The topography here is not periodic (it has a finite bandwidth of $\mathrm{O}(1 / L)$ ), but as $L$ becomes greater than $\lambda_{1}$, it begins to "look" periodic on the length scale of the wave (in a WKB sense).

More physically, waves are generated near the bottom, propagate upward, and reflect back down. The pressure field in the region where the reflected waves encounter the bottom will be different from that in an infinitely deep fluid (where the energy is continuously radiated upward). However, if this reflected wave encounters a flat bottom (small $L / \lambda_{1}$ ), the resulting power input is very similar to the infinite-depth value (since that region does not contribute to the work). On the other hand, if the downward propagating waves encounter a slopping bottom topography $\left(L / \lambda_{1}>1\right)$, the force and power characteristics will be modified as seen in Fig. 6. It is important to note that I could have chosen, say, a sequence of Gaussian bumps and obtained the same qualitative behavior.

The ratio $L / \lambda_{1}$ emerges as a fundamental parameter in the finite-depth theory. For large values of $L / \lambda_{1}$ (extended topography), mode conversion in a finite-depth ocean $\left(\mathscr{P}_{\mathrm{F}}\right)$ will be substantially smaller than in an infinitely deep ocean $\left(\mathscr{P}_{\infty}\right)$. Conversely, as $L / \lambda_{1} \rightarrow 0$ (isolated topography), $\mathscr{P}_{\mathrm{F}}$ will approach $\mathscr{P}_{\infty}$.

Having examined finite depth effects, I next discuss the effect of nonlinearities.

\section{Numerical calculations}

In this section, I present results of simulations of internal tides in a numerical model. In contrast to previous numerical investigations of internal tide generation in the open ocean (for e.g., Holloway and Merrifield, 1999; Merrifield et al., 2001), my goals are more process oriented. In particular, I will use the numerical results both to assess the theoretical analysis and to explore parameters for which the theory is formally invalid. To my knowledge, such a systematic comparison between theory and numerical calculations has not been previously attempted for internal tide generation.

\subsection{Model formulation}

To study the generation of internal waves by tidal flow over topography, I use the MIT general circulation model (GCM) (Marshall et al., 1997). The MIT GCM solves the nonhydrostatic, nonlinear primitive equations using a finite-volume formulation. Topography is represented by lopped cells (partial steps) (Adcroft et al., 1997). This feature is essential for accurately representing the interaction of the barotropic tide with the topography and hence the wave generation process. The partial step formulation also allows for more accurate diagnoses of topographic drag and power input, which are an important motivation for performing these calculations. The model has an implicit free-surface formulation.

To model a barotropic tide of amplitude $U_{0}$ and frequency $\omega_{0}$, a spatially uniform body force, $F_{\text {body }}=U_{0} \omega_{0} \cos \omega_{0} t$, is added to the zonal momentum equation. In the presence of topography and a time-dependent mean flow it no longer makes sense to decompose the motions into barotropic and baroclinic "modes" (although I continue to use the term "barotropic" in the sense of being independent of the vertical coordinate). Instead, "wave" motions are defined via,

$u^{\prime}(x, z, t) \equiv u(x, z, t)-U(x, t)$,

where $u$ is the zonal velocity, $U$ is defined as

$U(x, t) \equiv \frac{1}{H-h(x)} \int_{h(x)}^{H} u(x, z, t) \mathrm{d} z$

and $H$ is the nominal depth of the fluid layer and the bottom is at $z=h(x)$ (Fig. 2). I have found that $U(x, t)$ is practically independent of $x$, and is typically within a few percent of its desired value, $U_{0} \cos \omega_{0} t$.

An important issue in numerical solutions of wave problems is the treatment of open boundaries. Here, we would like to impose the requirement that the energy flux be outward from the generation site. This is the Sommerfeld radiation 
condition. This mathematical requirement was implicitly imposed when I solved the linear wave generation problem above (see, for example, Carrier et al., 1966). For consistency, radiation conditions (described in the appendix) have been implemented for the eastern and western open boundaries which permit the wave energy to leave the domain with minimal reflection. I have also found that radiation conditions (which are applied only to the "waves") work much better than "sponge layers" which tend to generate spurious reflections. In the meridional direction, periodic $\mathrm{BC}$ are imposed.

\subsection{Model parameters}

Numerical calculations using two idealized bottom topographies will be discussed: a truncated sine wave (wavelength fixed at $12 \mathrm{~km}$ and variable amplitude) similar to that discussed in the previous section (Fig. 4), and a Gaussian bump (variable width and amplitude). The horizontal and vertical grid spacing, $\Delta x$ and $\Delta z$, respectively, were adjusted to place the highest resolution near the topography. For the truncated sine, $\Delta x$ was fixed at $200 \mathrm{~m}$ near the obstacle $(60$ grid points per wavelength) and then gradually increased to $\approx 1500 \mathrm{~m}$ toward the boundaries (see Table 1). For the Gaussian bump experiments, horizontal resolution near the obstacle was varied according to the width of the bump which ranged from $500 \mathrm{~m}(\Delta x=20 \mathrm{~m})$ to $20 \mathrm{~km}(\Delta x=200 \mathrm{~m})$. In both cases, $\Delta z$ was fixed at $5 \mathrm{~m}$ near the topography and then gradually increased to $\approx 60 \mathrm{~m}$ near the surface. The nominal depth of the domain, $H$, was $4700 \mathrm{~m}$. The fluid is temperature stratified.

To compare the predictions of the inviscid theory with the numerical results, the diffusivity and viscosity were chosen so as to minimize explicit diffusion and dissipation. Note, however, that to maintain numerical stability, there must be a finite amount of explicit dissipation (in addition to numerical dissipation), and this should be kept in mind when comparing the numerical results with theory. In particular, a nonzero dissipation will enhance mode conversion relative to the inviscid theory.
Table 1

Model parameters for the numerical experiments

\begin{tabular}{ll}
\hline Parameter & Value \\
\hline$N$ & $8 \times 10^{-4} \mathrm{~s}^{-1}$ \\
$f$ & $8 \times 10^{-5} \mathrm{~s}^{-1}$ \\
$U_{0}$ & $2 \mathrm{~cm} \mathrm{~s}^{-1}$ \\
$\omega_{0}$ (nominal depth) & $1.4 \times 10^{-4} \mathrm{~s}^{-1}$ \\
$H$ (horizontal biharmonic viscosity) & $4700 \mathrm{~m}$ \\
$v_{\mathrm{H}}$ & $0.2-5 \times 10^{3} \mathrm{~m}^{4} \mathrm{~s}^{-1}$ \\
$\kappa_{\mathrm{H}}$ (horizontal biharmonic diffusivity) & $0.2-5 \times 10^{3} \mathrm{~m}^{4} \mathrm{~s}^{-1}$ \\
$v_{\mathrm{v}}$ (vertical viscosity) & $1 \times 10^{-5} \mathrm{~m}^{2} \mathrm{~s}^{-1}$ \\
$\kappa_{\mathrm{v}}$ (vertical diffusivity) & $1 \times 10^{-6} \mathrm{~m}^{2} \mathrm{~s}^{-1}$ \\
$\Delta t$ & $10-100 \mathrm{~s}$ \\
\hline
\end{tabular}

All calculations were initiated from a state of rest, and the model integrated for 20 forcing periods $\left(2 \pi / \omega_{0}\right)$. There was no noticeable erosion of the basic state stratification at the end of the integration. A steady state was generally achieved within 4-5 forcing periods. All diagnostics presented below represent suitable averages between forcing periods 8 and 12 .

\subsection{Diagnosing the force and energy flux}

The presence of an obstacle results in a substantial sea-surface height (SSH) response, which must be taken into account when the bottom pressure is computed. To separate the SSH signal due to the "background" flow, $U(t)$, from the (substantial) SSH response due to the internal tides, each model simulation was preceded by a "barotropic" simulation employing a single layer (i.e., no stratification). All other parameters were identical, and the instantaneous zonal velocity field in the barotropic calculation was the same as the background flow, $U(t)$, in the full model. The SSH response of the barotropic model, $\eta_{\mathrm{BT}}$, was subtracted from the $\mathrm{SSH}$ response of the full model, $\eta$, when the pressure was computed. The perturbation pressure, $p^{\prime}$, is thus defined as

$p^{\prime}(x, z, t) \equiv \rho_{0} g\left(\eta-\eta_{\mathrm{BT}}\right)+p(x, z, t)-P_{0}(z)$,

where, $p$ is the actual pressure field (not including the free-surface contribution), $\rho_{0}$ a reference density, $g=9.81 \mathrm{~m} \mathrm{~s}^{-2}$ the acceleration due to gravity, and $P_{0}(z)$, the hydrostatic reference 
pressure when the fluid is at rest. The force on the topography was then calculated according to

$F(t)=\int p^{\prime}(x, z=h(x), t) \frac{\mathrm{d} h}{\mathrm{~d} x} \mathrm{~d} x$,

where the (zonal) integration is over the entire domain. In numerically evaluating the integral I account for the topographic slope appearing in the integrand by exploiting the lopped cell representation of the bottom topography. The power per unit cross-stream length is then simply,

$\mathscr{P}_{\mathrm{N}} \equiv \frac{\omega_{0}}{2 \pi} \int_{0}^{2 \pi / \omega_{0}} F(t) U(t) \mathrm{d} t$.

It should be emphasized that including the contribution of the sea-surface displacement due to the internal tides to $p^{\prime}$ is crucial for accurately diagnosing the topographic force, and hence the power. This component of $p^{\prime}$ is of course the same as would be exerted by a "rigid lid", which is the assumption made in deriving the analytical solutions.

\subsection{Model results}

\subsubsection{Linear regime}

As a first step, I have used the numerical model to validate the linear, finite-depth theory derived above. A series of calculations were performed with the weak $(\varepsilon \approx 0.18)$ truncated sine topography of the previous section. Fig. 6 (circles) shows the power (normalized by $\mathscr{P}_{\infty}$ ) diagnosed in the numerical model. As discussed above, finite-depth theory predicts a substantially different energy flux compared to Bell's (1975a) infinite-depth solution. This prediction, and in particular the sharp decrease in mode conversion as $L / \lambda_{1}$ increases, is well reproduced in the model. Overall, there is very good agreement between the numerical calculations and the finite-depth theory.

\subsubsection{Nonlinear regime}

Linear theory is valid only for $\varepsilon$ much less than 1 , but the numerical model allows us to explore a more nonlinear regime. A series of model runs, with two different bottom topographies, were performed to examine how the power input varies with the steepness parameter, $\varepsilon$. Table 2 summarizes various parameters for the different experiments (labeled S-1, S-2, G-1, and G-2).

Truncated sine topography. Two sets of calculations were performed with this topography. The first set (S-1) has $L / \lambda_{1} \approx 0.19$, for which the finiteand infinite-depth theories predict the same power. The second set (S-2) has $L / \lambda_{1} \approx 1.7$, for which the two theories predict very different conversion rates (Fig. 6). In both $\mathrm{S}-1$ and $\mathrm{S}-2, \varepsilon$ was varied between 0.18 and 1.8 by varying $h_{0}$ between 50 and $500 \mathrm{~m}$. Figs. 7 (S-1) and 8 (S-2) show the zonal "wave" velocity, $u^{\prime}$, for $\varepsilon \approx 0.9\left(h_{0}=250 \mathrm{~m}\right)$. The topography is close to critical. In S-1, the length, $L$, of the obstacle is much smaller than the wavelength of the mode-1 wave and the response (Fig. 7, top panel) is distinctly "ray" like. Reflection from the free surface is clearly visible. In contrast, S-2 $\left(L>\lambda_{1}\right)$ displays a more "modal" structure in the vertical (Fig. 8, top panel) as predicted by the theory. (The dominant vertical mode excited will of course depend on the details of the bottom topography.) The bottom panels in Figs. 7 and 8 give a close-up view of $u^{\prime}$ near the topography. Note, that the amplitude of $u^{\prime}$ is much greater than that of the barotropic flow, $U\left(2 \mathrm{~cm} \mathrm{~s}^{-1}\right)$, and we should not expect linear theory to hold for these parameters. There is also much more vertical structure in the $u^{\prime}$ field just above the topography

Table 2

Characteristics of idealized bottom topographies used in the 4 numerical experiments

\begin{tabular}{lllll}
\hline Experiment & Topography & $L / \lambda_{1}$ & $\varepsilon \sim h_{0} / \lambda$ & Parameters \\
\hline S-1 & Truncated sine: $h_{0} \sin (2 \pi x / \lambda)$ & 0.19 & $0.18-1.8$ & $\lambda=12 \mathrm{~km}($ fixed $) ; h_{0}(50-500 \mathrm{~m})$ \\
S-2 & Truncated sine: $h_{0} \sin (2 \pi x / \lambda)$ & 1.7 & $0.18-1.8$ & $\lambda=12 \mathrm{~km}($ fixed $) ; h_{0}(50-500 \mathrm{~m})$ \\
G-1 & Gaussian bump: $h_{0} \exp \left(-x^{2} / 2 \lambda^{2}\right)$ & $0.62-0.016$ & $0.04-1.65$ & $h_{0}=200 \mathrm{~m}($ fixed $) ; \lambda(20 \mathrm{~km}-500 \mathrm{~m})$ \\
G-2 & Gaussian bump: $h_{0} \exp \left(-x^{2} / 2 \lambda^{2}\right)$ & 0.19 & $0.04-1.6$ & $\lambda=6 \mathrm{~km}($ fixed $) ; h_{0}(50-2300 \mathrm{~m})$ \\
\hline
\end{tabular}



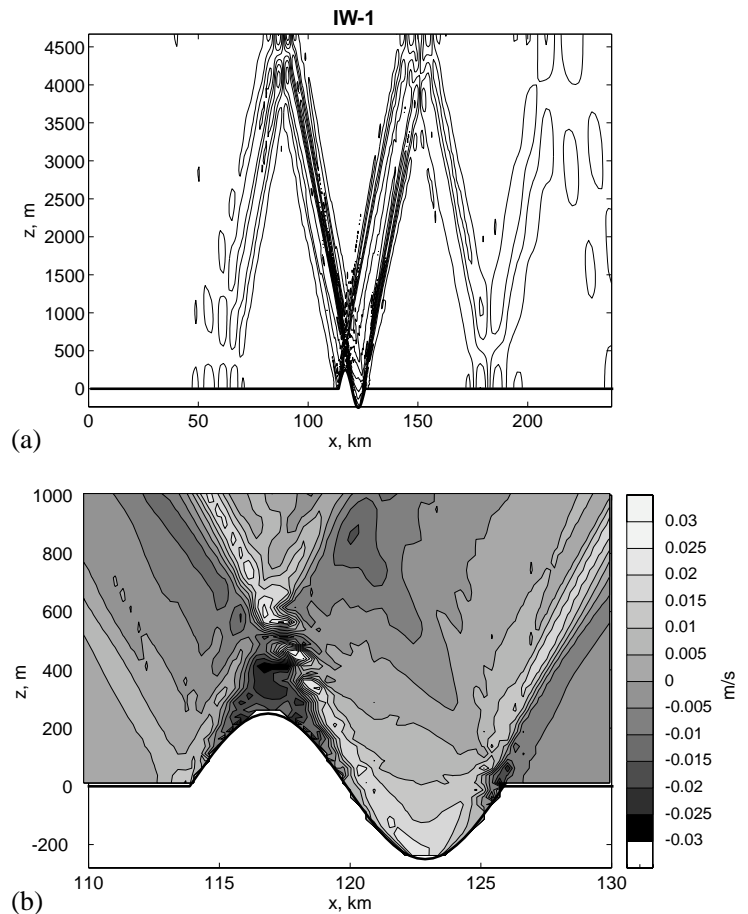

(b)

Fig. 7. Contour plots of horizontal wave velocity, $u^{\prime}$, in S-1 $(\varepsilon \approx 0.9)$. Contours range from -3 to $+3 \mathrm{~cm} \mathrm{~s}^{-1}$. The bottom panel gives an expanded view of the $u^{\prime}$ field near the topography.

than is predicted by linear theory (the fine scale features are likely a result of the numerics, and in particular the very low diffusivity and viscosity). We might thus expect the vertical shear in this region to lead to wave breaking, although no overturning events have been observed in these calculations. For steeper topography, $u^{\prime}$ near the topography was found to be as large as 10 $12 \mathrm{~cm} \mathrm{~s}^{-1}$.

Figs. 9 and 10 show the power diagnosed in experiments $\mathrm{S}-1$ and $\mathrm{S}-2$ as a function of $\varepsilon$. Also shown is the conversion rate predicted by finitedepth theory. All values are normalized by $\mathscr{P}_{\infty}$. It is interesting to note that the ratio $\mathscr{P}_{\mathrm{F}} / \mathscr{P}_{\infty}$ remains constant in both $\mathrm{S}-1$ and $\mathrm{S}-2$, suggesting that the finite-depth power can be written as $\mathscr{P}_{\mathrm{F}}=$ $\mathscr{P}_{\infty} \times f\left(L / \lambda_{1}\right)$, where the specific form of $f$ will depend on the topography. Llewellyn Smith and Young (2002) have made a similar observation,
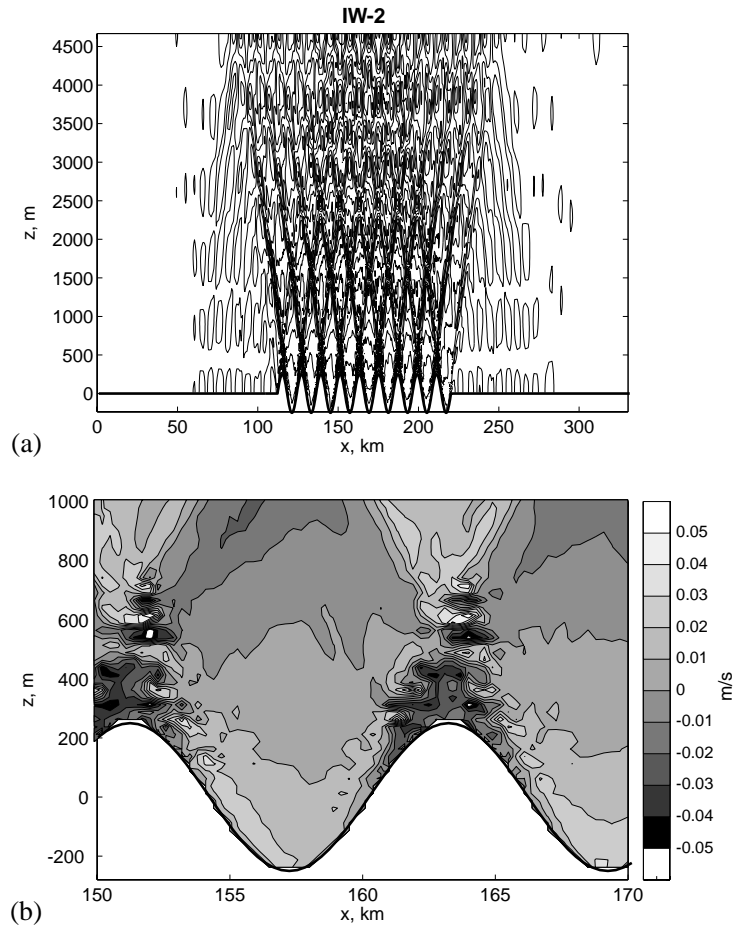

Fig. 8. Contour plots of horizontal wave velocity, $u^{\prime}$, in $\mathrm{S}-2$ $(\varepsilon \approx 0.9)$. Contours range from -5 to $+5 \mathrm{~cm} \mathrm{~s}^{-1}$. The bottom panel gives an expanded view of the $u^{\prime}$ field near the topography.

and have also derived the form of $f$ for some specific topographic shapes.

In both $\mathrm{S}-1$ and $\mathrm{S}-2$, the mode conversion rate predicted by linear theory is a monotonically increasing function of $\varepsilon$ (see inset for absolute value of power per unit area). At first, the numerical power also increases with $\varepsilon$, and at a faster rate compared to theory. In S-1, at critical slope $(\varepsilon=1)$, finite-depth and infinite-depth theories both underestimate mode conversion by $\approx 20 \%$ relative to the numerical model. In $\mathrm{S}-2$, the corresponding underestimates are about $90 \%$ and $6 \%$, respectively. (In S-2, for $\varepsilon \ll 1$, the numerical value is in better agreement with the finite-depth theory.) As the topography becomes supercritical $(\varepsilon>1)$, the numerical power decreases relative to the theoretical value. Note, though, that the absolute power per unit area (inset) saturates and remains roughly constant with $\varepsilon$. 


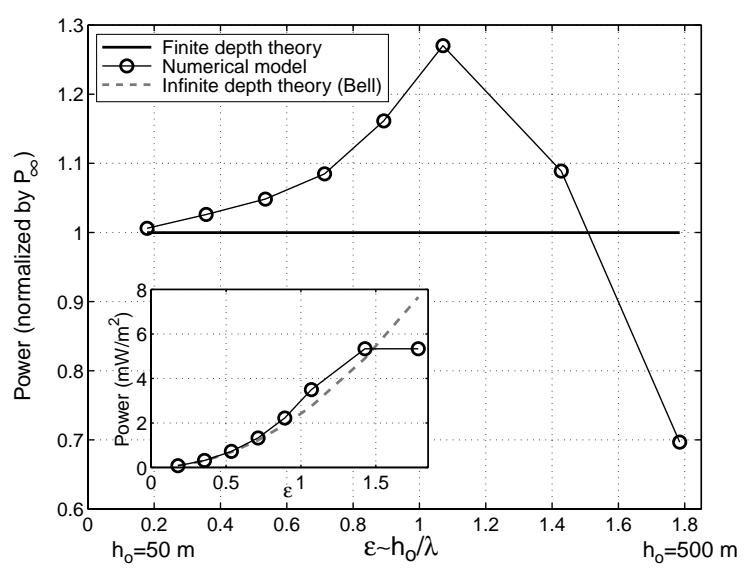

Fig. 9. Comparison of power input to the internal wave field diagnosed in numerical simulations (S-1) with predictions of finite- and infinite-depth linear theories. The conversion rates are normalized by $\mathscr{P}_{\infty}$ and plotted against the steepness parameter, $\varepsilon$. For reference, the inset shows the absolute infinite-depth conversion rate (per unit area). As discussed in the text, the absolute conversion rate in the numerical model saturates at a roughly constant value when the topography becomes supercritical.

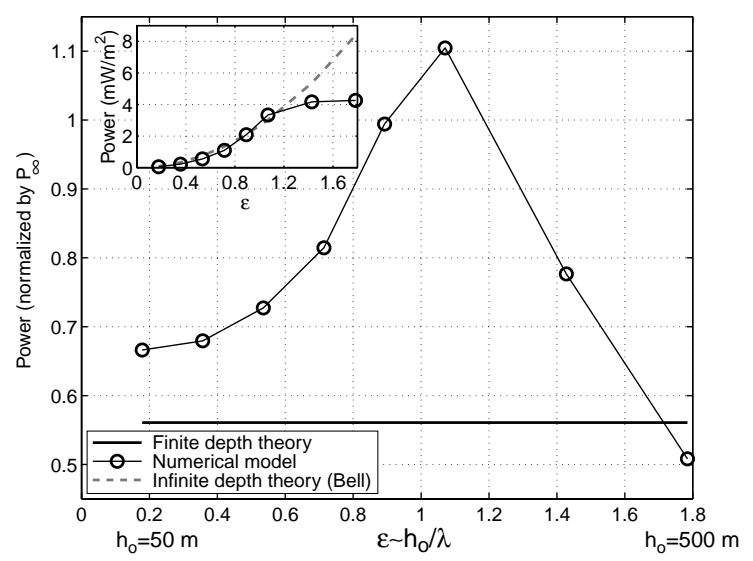

Fig. 10. Same as Fig. 9, but for S-2.

The saturation of power for supercritical topography is an important finding of this study. It was initially suspected that power saturation was caused by a decrease in the stratification. As shown by Balmforth et al. (2002), in a linear, inviscid theory, as $\varepsilon \rightarrow 1$, the wave field develops very small spatial scales, and when $\varepsilon=1$ the solutions formally become singular. (Retaining advection by the basic state in the governing equations may change this behavior, although this remains to be seen.) The progressively smaller vertical scales in the wave field as the topography became steeper could lead to overturning and mixing. However, the diagnosed Richardson number in the model was always large, and no overturning events were observed. Furthermore, the stratification did not discernibly change from its initial value over the course of the integration. (Balmforth et al. suggest that physical processes such as nonlinearity and dissipation, which are both present in the numerical model, may be required to remove the singularity at the transition $\varepsilon=1$.) This raises the question of whether the saturation effect is peculiar to this topography or the manner in which $\varepsilon$ is changed. For instance, it is possible that in the nonlinear regime the amplitude of the topography relative to the mean depth of the fluid plays a role in halting the power increase. To investigate this, $\varepsilon$ could be varied by changing the wavelength $(\lambda)$ of the topography, while keeping its amplitude fixed. This could be done, but interpreting the results would not be entirely straightforward. This is because $L$ is related to $\lambda(L=m \lambda)$ and, as shown above, any changes in the ratio $L / \lambda_{1}$ will also modify the energetics of the wave generation. Alternatively, if we insist on keeping $L$ fixed, the number of sines, $m$, must correspondingly be increased as $\lambda$ is decreased. This both increases the computational expense and introduces its own complications because of reflection and interference of multiple rays. To avoid these complications and address the issue of power saturation, two additional sets of calculations were performed with a different bottom topography, namely a Gaussian bump.

Gaussian topography. Results from experiments with a Gaussian bump, G-1 and G-2, are shown in Figs. 11 and 12. In G-1, the height was fixed at $200 \mathrm{~m}$, and the half-width, $\lambda$ varied between $20 \mathrm{~km}$ and $500 \mathrm{~m}$ ( $\varepsilon$ ranges between 0.04 and 1.65). In G2 , the half-width was fixed at $6 \mathrm{~km}$, and the height increased from 50 to $2300 \mathrm{~m}$ ( $\varepsilon$ varies from 0.04 to 1.6). Note that in G-1 the ratio $L / \lambda_{1}$ is not constant and varies with $\varepsilon(\sim 1 / L)$. For $\varepsilon \ll 1$, the finite depth has a significant influence on the energetics $\left(\mathscr{P}_{\mathrm{F}} \ll \mathscr{P}_{\infty}\right)$, an effect also captured by the numerical model. 


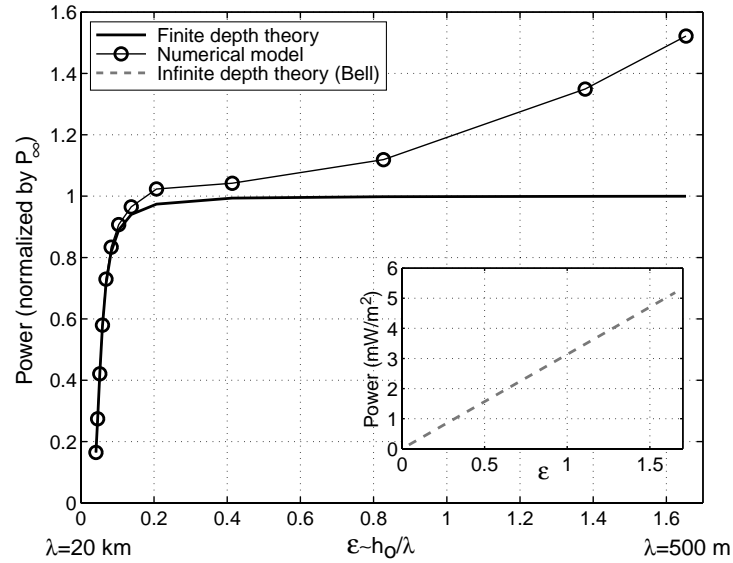

Fig. 11. Same as Fig. 9, but for G-1. In contrast to the truncated sine case, the conversion rate in the numerical model does not saturate for $\varepsilon>1$.

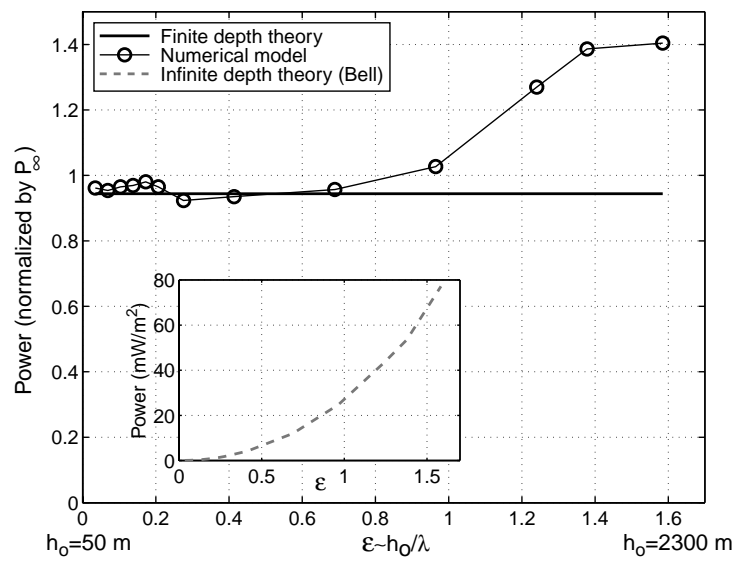

Fig. 12. Same as Fig. 9, but for G-2. As in G-1, the conversion rate in the numerical model continues to increase with $\varepsilon$ even for supercritical topography.

As in the previous experiments, linear theory underestimates the mode conversion rate (by $\approx 10-20 \%$ ). There is, however, a significant difference between these calculations and the previous ones, in that with a Gaussian topography there is no evidence of power saturation as the topography becomes supercritical. This is true regardless of the manner in which $\varepsilon$ is changed. The most straightforward explanation for this difference is the trapping of energy in the troughs of the sinusoidal topography: once the topography is supercritical, there is downward energy propa- gation. No such effect is possible with a single Gaussian bump (although a set of Gaussian bumps should qualitatively display the same behavior as the sinusoidal topography).

Comparison with a finite amplitude theory. Balmforth et al. (2002) (hereafter BIY) have recently developed an analytical theory of internal tide generation in a fluid of infinite depth which is valid for $0 \leqslant \varepsilon \leqslant 1$. Applying this theory to a sinusoidal topography and a Gaussian bump they find that at critical slope $(\varepsilon=1)$ mode conversion is enhanced (relative to linear, infinite-depth theory) by $56 \%$ and $14 \%$, respectively. The latter value is comparable to the enhancement factors observed in G-1 and G-2 (10-20\%), although it is important to recognize that the BIY theory is valid only for an infinitely deep fluid. In both G-1 and G-2, the ratio $L / \lambda_{1}$ is small, and from the point of view of a linear theory, the fluid is effectively of infinite depth $\left(\mathscr{P}_{\mathrm{F}} \approx \mathscr{P}_{\infty}\right)$, so arguably a comparison with the BIY theory is not unreasonable. Although this is also the case in experiment S-1, the topography there is a truncated sine and not an infinite sinusoidal one for which linear, finitedepth theory predicts zero conversion. Thus it is not clear whether a comparison between S-1 and the results of BIY is justified. Nonetheless, the numerical experiments presented here give an enhancement of $\approx 20 \%$, which is much smaller than the $56 \%$ enhancement predicted by BIY. One possible explanation for this difference is as follows. Linear theory showed that when the parameter $L / \lambda_{1 \mid}$ is small, the fluid is effectively of infinite depth. For their theory to be applicable, BIY assume that this remains true even when the topography is of finite amplitude. But the numerical results (to the extent they can be compared with BIY) suggest that even though finite-depth effects may be negligible in the linear regime, they may become important when the topography is of finite amplitude.

\section{Conclusions}

In this paper, analytical and numerical techniques have been applied to better understand the process of internal tide generation in the ocean by 
the interaction of the barotropic tide with bottom topography. A new analytical theory has been developed for the generation of internal tides in an ocean of finite depth. Previous theoretical studies of internal tide generation in the ocean have often assumed that dissipation processes are sufficiently strong to justify treating the ocean as a fluid of infinite depth (Bell, 1975b). Mathematically, this is equivalent to applying an upper radiation BC. However, recent observations indicate that reflection of wave energy from the upper surface is significant, and therefore treating the ocean as a fluid of infinite depth is not always justified. I have shown here that the presence of an upper reflecting surface can significantly reduce, relative to the infinite-depth theory of (Bell 1975a), the rate of transfer of barotropic tidal energy into the internal wave field. This reduction is particularly large for extended topographic features.

I have also compared the power input predicted by linear theory with the conversion rate diagnosed in a nonhydrostatic, nonlinear, hydrodynamic model. Two model topographies, a truncated sine and a Gaussian bump, are used to explore the internal tide response. There is good agreement between the theory and model for small values of the steepness parameter $\varepsilon$ for which the theory is formally valid. As $\varepsilon$ becomes $\mathrm{O}(1)$, linear theory underestimates the power by an increasing amount, although the theory still provides reasonably accurate estimates of mode conversion. As the topography becomes supercritical, the power saturates for the truncated sine topography, but not the Gaussian bump.

\section{Acknowledgements}

I would like to thank Carl Wunsch, Sonya Legg, and Kurt Polzin for innumerable discussion on internal waves and for making many helpful comments on the manuscript. Joe Pedlosky patiently advised on various mathematical issues. I am grateful to Alistair Adcroft and Jean-Michel Campin for their assistance with the numerical calculations. Discussions with Bill Young and Lou St. Laurent and the comments of 3 anonymous referees helped improve the text. This work was supported by a William Z. Leavitt Postdoctoral Fellowship at MIT.

\section{Appendix A. Implementation of radiation boundary conditions}

The implementation of the radiation $\mathrm{BCs}$ is based on that proposed by Orlanski (1976). Consider a variable $\phi$ governed by the hyperbolic equation

$\frac{\partial \phi}{\partial t}+C \frac{\partial \phi}{\partial x}=0$

where $C$ is the phase speed. Eq. (A.1) carries information about $\phi$ in the $+x$ direction if $C>0$ and the $-x$ direction if $C<0$. The waves we are numerically modeling are much more complicated and in general their dispersion properties are not known either. To practically implement the Sommerfeld radiation condition, Orlanski (1976) proposed using an equation of the form (A.1) to predict the value of model variables on open boundaries. The phase speed, $C$, is different for each variable and is to be diagnosed from the tendency and spatial gradients of the model fields as

$C_{\phi}=\frac{-\partial \phi / \partial t}{\partial \phi / \partial x}$

Given a $C_{\phi}$, Eq. (A.1) can be used to prognostically determine values on the open boundary.

The actual numerical implementation of the above ideas must be consistent with the specific time-stepping scheme used in the model. In the MIT GCM, this is a $2 \mathrm{~d}$ order Adams-Bashforth scheme (AB-II). As example, I give the discretization for an eastern open boundary (a subscript is the zonal index; a superscript the time step). For each grid point along the eastern boundary in the meridional and vertical directions we have

$C_{B-1}^{n}=\frac{-\left[\phi_{B-1}^{n}-\phi_{B-1}^{n-1}\right]}{\left[a_{1}\left(\phi_{B-1}^{n-1}-\phi_{B-2}^{n-1}\right)+a_{2}\left(\phi_{B-1}^{n-2}-\phi_{B-2}^{n-2}\right)\right]} \frac{\Delta x}{\Delta t}$,

where $B$ is the boundary grid point, $\Delta x$ the horizontal resolution and $\Delta t$ the time step, $a_{1}=$ $1.5+\varepsilon$ and $a_{2}=-0.5-\varepsilon$ are AB-II coefficients ( $\varepsilon$ is a small number). For stability reasons, $C_{\phi}$ is not allowed to exceed the value $0.5 \Delta x / \Delta t$. (The 
prefactor, 0.5, is due to the more stringent Courant condition on the AB-II scheme). In addition, if $C_{B-1}^{n}<0, C_{B-1}^{n}$ is set equal to 0 . The value of $\phi$ on the open boundary is then calculated according to

$$
\begin{aligned}
\phi_{B}^{n+1}= & \phi_{B}^{n}-C_{B-1}^{n} \frac{\Delta t}{\Delta x} \\
& \times\left[a_{1}\left(\phi_{B}^{n}-\phi_{B-1}^{n}\right)+a_{2}\left(\phi_{B}^{n-1}-\phi_{B-1}^{n-1}\right)\right] .
\end{aligned}
$$

I have found that the scheme just described is quite prone to numerical instability and also gives rise to spurious reflections. Apparently, the diagnosed phase speed fluctuates in time and frequently exceeds the allowable maximum speed (to which it is then clamped). A simple way to overcome this difficulty is to low-pass filter (in time) the phase speed. This is implemented as

$$
C^{n} \equiv \frac{\Delta t}{T} C^{n} \text { (diagnosed) }+\left(1-\frac{\Delta t}{T}\right) C^{n-1},
$$

where $T$ is an averaging period. Setting $T$ to the period of the dominant waves appears to work very well.

\section{References}

Adcroft, A., Hill, C., Marshall, J., 1997. Representation of topography by shaved cells in a height coordinate ocean model. Monthly Weather Review 125, 2293-2315.

Baines, P.G., 1973. The generation of internal tides by flatbump topography. Deep-Sea Research 20, 179-205.

Baines, P.G., 1982. On internal tide generation models. DeepSea Research 29, 307-338.

Baines, P.G., 1995. Topographic Effects in Stratified Flows. Cambridge Monographs on Mechanics. Cambridge University Press, Cambridge.

Balmforth, N.J., Ierley, G.R., Young, W.R., 2002. Tidal conversion by nearly critical topography. Journal of Physical Oceanography 32, 2900-2914.

Bell, T.H., 1975a. Lee waves in stratified flows with simple harmonic time dependence. Journal of Fluid Mechanics 67, 705-722.

Bell, T.H., 1975b. Topographically generated internal waves in the open ocean. Journal of Geophysical Research 80, 320-327.

Carrier, G.F., Krook, M., Pearson, C.E., 1966. Functions of a Complex Variable. McGraw-Hill Book Company, Inc., New York.

Dushaw, B.D., Howe, B.M., Cornuelle, B.D., Worcester, P.F., Luther, D.S., 1995. Barotropic and baroclinic tides in the central North Pacific Ocean determined from long-range reciprocal acoustic transmissions. Journal of Physical Oceanography 25, 631-648.
Egbert, G.D., Ray, R.D., 2000. Significant dissipation of tidal energy in the deep ocean inferred from satellite altimeter data. Nature 405, 775-778.

Gill, A.E., 1982. Atmosphere-Ocean Dynamics. Academic Press, New York.

Hibiya, T., 1986. Generation mechanism of internal waves by tidal flow over a sill. Journal of Geophysical Research 91, 7697-7708.

Holloway, P.E., Merrifield, M.A., 1999. Internal tide generation by seamounts, ridges, and islands. Journal of Geophysical Research 104, 25 937-25951.

Jayne, S.R., St. Laurent, L.C., 2001. Parameterizing tidal dissipation over rough topography. Geophysical Research Letters 28, 811-814.

Ledwell, J.R., Montgomery, E.T., Polzin, K.L., St. Laurent, L.C., Schmitt, R.W., Toole, J.M., 2000. Evidence for enhanced mixing over rough topography in the abyssal ocean. Nature 403, 179-182.

Llewellyn Smith, S.G., Young, W.R., 2002. Conversion of the barotropic tide. Journal of Physical Oceanography 32, 1554-1566.

Marshall, J., Adcroft, A., Hill, C., Perelman, L., Heisey, C., 1997. A finite-volume, incompressible Navier-Stokes model for studies of the ocean on parallel computers. Journal of Geophysical Research 102, 5733-5752.

McIntyre, M.E., 1972. On Long's hypothesis of no upstream influence in uniformly stratified rotating flow. Journal of Fluid Mechanics 52, 209-243.

Merrifield, M.A., Holloway, P.E., Johnston, T.M.S., 2001. The generation of internal tides at the Hawaiian Ridge. Geophysical Research Letters 28, 559-562.

Munk, W., 1997. Once again: once again-tidal friction. Progress in Oceanography 40, 7-35.

Munk, W., Wunsch, C., 1998. Abyssal recipes II: energetics of tidal and wind mixing. Deep-Sea Research I 45, 1977-2010.

Orlanski, I., 1976. A simple boundary condition for unbounded hyperbolic flows. Journal of Computational Physics 21, 251-269.

Polzin, K.L. Idealized solutions for the energy balance of the finescale internal wavefield. Journal of Physical Oceanography, submitted for publication.

Polzin, K.L., Toole, J.M., Ledwell, J.R., Schmitt, R.W., 1997. Spatial variability of turbulent mixing in the abyssal ocean. Science 276, 93-96.

Ray, R.D., Mitchum, G.T., 1996. Surface manifestation of internal tides generated near Hawaii. Geophysical Research Letters 23, 2101-2104.

St. Laurent, L.C., Garrett, C.J.R., 2002. The role of internal tides in mixing the deep ocean. Journal of Physical Oceanography 32, 2882-2899.

Watson, G.N., 1966. A Treatise on the Theory of Bessel Functions, 2nd Edition. Cambridge University Press, Cambridge.

Wunsch, C., 1998. The work done by the wind on the ocean circulation. Journal of Physical Oceanography 28, 2331-2339. 